\title{
DIABETES
}

\section{Genetically engineered Lactobacilli reprogram intestinal cells to secrete insulin and ameliorate hyperglycaemia}

Oral administration of Lactobacilli engineered to secrete the inactive fulllength form of glucagon-like peptide 1 (GLP- $1_{1-37}$ ) markedly reduces blood glucose levels, according to a new study in rats. The findings have potential implications for the treatment of patients with type 1 diabetes mellitus (T1DM).

Numerous strategies to replace $\beta$ cells destroyed by autoimmune attack in T1DM have been proposed, including the reprogramming of pancreatic non- $\beta$ cells

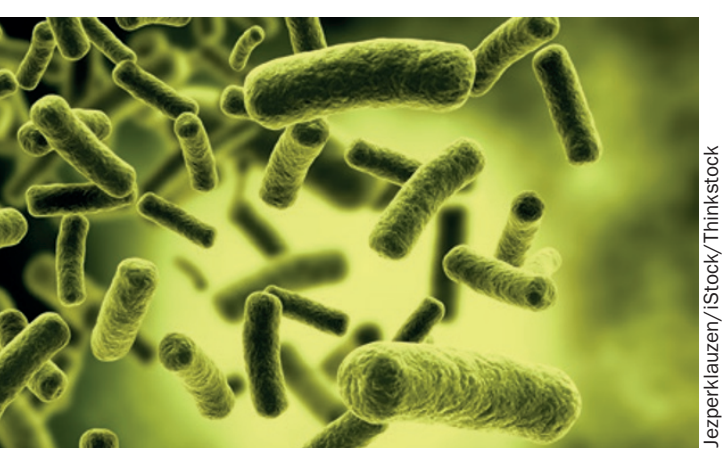

and other tissue-specific cells into $\beta$ cells or cells with insulin-secreting potential. Previously, the researchers showed that genetically engineered commensal bacteria could deliver GLP-1 ${ }_{1-37}$ to human intestinal carcinomas and stimulate glucose-responsive insulin secretion. In the current study the investigators tested whether similar bacteria could reduce hyperglycaemia in rats with streptozotocin-induced T1DM. "Our goal was to reprogram rat intestinal cells into glucose-responsive insulin-secreting cells through daily oral administration of GLP- $1_{1-37}$-secreting bacteria," explain the authors in their report.

Diabetic rats fed GLP- $1_{1-37}$-secreting Lactobacilli daily for 50 days had higher levels of insulin and were more glucosetolerant following an oral glucose tolerance test than those fed wild-type Lactobacilli; no significant difference was observed in levels of blood glucose and plasma insulin between diabetic rats fed genetically engineered Lactobacilli and nondiabetic control rats. Reduced hyperglycaemia in rats fed GLP- $1_{1-37^{-}}$ secreting Lactobacilli was accompanied by the appearance of insulin-secreting intestinal epithelial cells that expressed key $\beta$-cell markers (pancreas/duodenum homeobox protein 1 , transcription factor MafA and forkhead box protein A2) indicative of reprogramming to an insulin-producing phenotype.

"These results provide evidence of the potential for a safe and effective nonabsorbed oral treatment for diabetes [mellitus] and support the concept of engineered commensal bacterial signalling to mediate enteric cell function in vivo," the authors conclude in their report.

\section{David Holmes}

Original article Duan, F. F. et al. Engineered commensal
bacteria reprogram intestinal cells into glucose-responsive
insulin-secreting cells for the treatment of diabetes.
Diabetes doi:10.2337/db14-0635




\section{CORRECTION}

Diabetes: Genetically engineered Lactobacilli reprogram intestinal cells to secrete insulin and ameliorate hyperglycaemia

David Holmes

Nat. Rev. Endocrinol. advance online publication 17 February 2015; doi:10.1038/nrendo.2015.45

This article was originally published with the incorrect doi. Instead of 10.1038/

nrendo.2015.14, the doi should have been 10.1038/nrendo.2015.45. This error has been corrected online in the HTML and PDF versions, and for the print issue. 